Bull. Austral. Math. Soc.

Vol. 45 (1992) [369-376]

\title{
THE GAP BETWEEN SUBSPACES AND PERTURBATION OF NON-SEMI-FREDHOLM OPERATORS
}

\author{
Jose A. Alvarez, Teresa Alvarez and Manuel Gonzalez
}

\begin{abstract}
We study a concept of stability under the gap of isomorphic properties of Banach spaces and apply it to obtain some results of stability under compact or small norm perturbation for non-semi-Fredholm operators with closed range.
\end{abstract}

\section{INTRODUCTION}

A classical result of Fredholm theory states that perturbing a semi-Fredholm operator $T$, (that is, an operator with closed range $R(T)$ and finite dimensional kernel $N(T)$ or cokernel $Y / R(T))$ by an operator $A$ with norm $\|A\|<\gamma(T)$, the minimum modulus of $T$, or $A$ compact, we obtain a semi-Fredholm operator $T+A$ with $\operatorname{dim} N(T+A) \leqslant \operatorname{dim} N(T)$ and $\operatorname{dim} Y / R(T+A) \leqslant \operatorname{dim} Y / R(T)$. Hence, for semiFredholm operators the finite dimension of the kernel or the cokernel is stable under small or compact perturbations. However, for non-semi-Fredholm operators, we have the opposite case: Given such an operator we can find a compact operator with arbitrarily small norm such that the perturbed operator has infinite dimensional kernel and cokernel [6], (see also [7]).

In [1] Bouldin divided the class NSF of bounded non-semi-Fredholm operators, acting in a separable Hilbert space, into six disjoint subclasses $U_{i}(i=0,1,2,3,4,5)$. He proved that for $i \leqslant 4$ the subclasses are totally unstable: $U_{i}+C o=N S F$, where $C o$ denotes the class of compact operators. However, the class $U_{5}$, the class of all operators with closed range and infinite dimensional kernel and cokernel, is not totally unstable. Later, Gonzalez and Onieva [7] studied the problem for bounded operators in Banach spaces, obtaining the same results for $U_{i}(i \leqslant 4)$. These results were extended by Labuschagne [13] to closed operators acting in operator ranges. However, in these papers the stability of $U_{5}$ is not investigated.

On the other hand, the gap between two closed subspaces of a Banach space, introduced to study the perturbation of closed semi-Fredholm operators (see [12]), has been used by several authors $[2,10,15]$ to analyse the preservation of certain isomorphic properties of a subspace $M$ of a Banach space (separability, reflexivity, containing no

Received 30 April 1991

Supported in part by DGICYT grant PB88-0417

Copyright Clearance Centre, Inc. Serial-fee code: 0004-9729/92 \$A2.00+0.00. 
copies of $\ell_{1}, \ldots$ ) when one passes from $M$ to another subspace which is close to $M$ in the sense of the gap.

In this paper we introduce the concept of stability of an isomorphic class of Banach spaces with respect to the non-symmetric gap $\delta(M, N)$ (which is the correct one for our purposes), we study the properties of stable classes, and we provide several examples and counterexamples. Parts of the results are analogous to those of $[\mathbf{2}, \mathbf{1 0}, \mathbf{1 5}]$, obtained for the symmetric gap $g(M, N):=\max \{\delta(M, N), \delta(N, M)\}$, but we also prove some new ones. For example, if $P$ is a stable class, then $\left\{X: X^{* *} / X \in P\right\}$ is also stable; and this shows the stability of several classes.

Next, using the results of stability for subspaces, we show that the corresponding properties of the kernel or the cokernel of a closed operator with closed range are stable under compact or small norm perturbations, and some subsets of $U_{5}$ are not totally unstable under compact perturbation.

Notations. Given closed subspaces $M$ and $N$ of a Banach space $X$, we denote by $M^{0}$ the annihilator of $M$ in the dual space $X^{*}$. Moreover, tha gap $\delta(M, N)$ between $M$ and $N[10,11]$ is defined by

$$
\delta(M, N):=\sup \{\operatorname{dist}(m, N): m \in M,\|m\|=1\} .
$$

$C(X, Y)$ and $L(X, Y)$ will denote the classes of closed and bounded linear operators from $X$ into $Y$. For $T \in C(X, Y), D(T), N(T)$ and $R(T)$ will be the domain, the kernel and the range of $T$, and $\gamma(T)$ will denote the minimum modulus of $T$ [5, 9], given by

$$
\gamma(T):=\inf \{\|T x\|: x \in D(T), \operatorname{dist}(x, N(T))=1\} .
$$

For $T \in C(X, Y), \gamma(T)>0$ if and only if $R(T)$ is closed [5, IV.1.6].

For an operator $T: X \rightarrow Y$ with domain $D(T)$ dense, $T^{*}: Y^{*} \rightarrow X^{*}$ will be the conjugate of $T[\mathbf{5}]$.

\section{STABLE PROPERTIES}

Let $P$ denote a class of Banach spaces stable under isomorphisms. We will say that a Banach space $X$ has property $P$ when $X \in P$.

Definition 2.1: Let $0<a \leqslant 1$.

$P$ is a-stable if for every Banach space $X$ and closed subspaces $M, N$ of $X$ we have

$$
N \in P, \quad \delta(M, N)<a \Rightarrow M \in P .
$$

$P$ is a-costable if for every Banach space $X$ and closed subspaces $M, N$ of $X$ we have

$$
X / M \in P, \quad \delta(M, N)<a \Rightarrow X / N \in P .
$$


OBSERvation 2.2: Since $\delta(M, N)=\delta\left(N^{0}, M^{0}\right)\left[11\right.$, IV.2.9] and $(X / M)^{*} \equiv M^{0}$, it is immediate that $P$ is $a$-stable ( $a$-costable) if and only if the dual class $P^{d}:=$ $\left\{X: X^{*} \in P\right\}$ is $a$-costable (a-stable).

Next we show some examples of $a$-stable and $a$-costable classes.

Examples 2.3: (a) The class of finite dimensional spaces is 1-stable.

(b) The classes of superreflexive, containing no copies of $\ell_{1}$ and $B$-convex Banach spaces are 1-stable (see $[15]$ and $[10,3.2 .10]$ ).

(c) The class of separable spaces is $1 / 2$-stable and $1 / 2$-costable.

Proof: (a) If $M, N$ are closed subspaces of $X$, then $\delta(M, N)<1$ implies $\operatorname{dim} M \leqslant \operatorname{dim} N$ [11, IV.2.6].

(b) We give the proof for the class of Banach spaces containing no copies of $\ell_{1}$.

Let $M, N$ be closed subspaces of a Banach space $X$, and suppose that $M$ contains a copy of $\ell_{1}$ and $\delta:=\delta(M, N)<1$.

By a result of James [8], $M$ contains an almost isometric copy of $\ell_{1}$. In particular, taking $\alpha>0$ such that $\delta<1-2 \alpha$, we can find a normalised sequence $\left(x_{n}\right)$ in $M$ so that $\left\|a_{1} x_{1}+\ldots+a_{n} x_{n}\right\| \geqslant(1-\alpha)\left(\left|a_{1}\right|+\ldots+\left|a_{n}\right|\right)$ for any scalars $a_{1}, \ldots, a_{n}$.

Now we can select $y_{i} \in N$ such that $\left\|x_{i}-y_{i}\right\|<1-2 \alpha$ for $i=1, \ldots, n, \ldots$; then we have $\left\|a_{1} y_{1}+\ldots+a_{n} y_{n}\right\| \geqslant \alpha\left(\left|a_{1}\right|+\ldots+\left|a_{n}\right|\right)$ for any scalars $a_{1}, \ldots a_{n}$, hence $N$ contains a copy of $\ell_{1}$.

The proof for the classes of superreflexive and $B$-convex spaces is analogous, using characterisations given in [9] and [4], respectively.

(c) Using a denseness argument, it is easy to prove that the class of separable spaces is $1 / 2$-stable.

On the other hand, suppose $X / M$ is separable and let $\varepsilon>0$ such that $\delta:=$ $\delta(M, N)<1 / 2-\varepsilon$. We shall construct a separable subspace $Z$ of $X$ such that every $x \in X$ with $\|x\|=1$ admits a decomposition $x=m+z$ with $m \in M, z \in Z$, and $\|z\|<1+\varepsilon$.

Let $\left(y_{n}\right)$ be a dense sequence in the unit sphere of $X / M$. Denoting by $q_{M}$ the quotient map onto $X / M$, there exist $0<t_{1} \leqslant 1$ and $n_{1}$ such that $\left\|q_{M} x-t_{1} y_{n_{1}}\right\|<$ $\varepsilon / 4^{2}$. Thus there are $0<t_{2} \leqslant \varepsilon / 4$ and $n_{2}$ such that $\left\|q_{M} x-t_{1} y_{n_{1}}-t_{2} y_{n_{2}}\right\|<\varepsilon / 4^{3}$. In this manner we obtain a series $\sum t_{i} y_{n_{i}}$ which converges to $q_{M} x$. Now, choosing $x_{i} \in X$ such that $y_{i}=q_{M} x_{i}$ and $\left\|x_{i}\right\|<1+\varepsilon / 2$, and taking as $Z$ the subspace generated by $\left(x_{n}\right)$, we have $x-\sum t_{i} x_{n_{i}} \in M$ and $\left\|\sum t_{i} x_{n_{i}}\right\|<1+\varepsilon$.

Now, if $X / N$ is not separable, then $N+Z$ is not dense in $X$, and hence there exists $x \in X,\|x\|=1$ such that $\operatorname{dist}(x, N+Z)>1-\varepsilon$. We can write $x=m+z$, $m \in M, z \in Z,\|z\|<1+\varepsilon$. Then choosing $n \in N$ such that $\|(m /\|m\|)-n\|<1 / 2-\varepsilon$ we deduce that $\|x-z-\| m\|n\|<1-\varepsilon$ which contradicts $\operatorname{dist}(x, N+Z)>1-\varepsilon$. 
Consequently $X / N$ is separable.

It is a surprising fact that a property is stable if and only if it is costable. This is shown in the next theorem.

Theorem 2.4. (1) If $P$ is a-costable then $P$ is a/2-stable.

(2) If $P$ is a-stable then $P$ is a/2-costable.

Proof: Let $X$ be a Banach space, and $M, N$ closed subspaces of $X$ for which $\delta=\delta(M, N)>0$.

(1) We denote by $E$ the Banach space $M \times N$ with the norm

$$
\|(x, y)\|_{E}:=\max \left\{\|x\|,\|y\|, \delta^{-1}\|x-y\|\right\}
$$

Consider the maps $U, V \in L(E, X)$ given by $U(x, y):=x ; V(x, y):=y$. Obviously $R(U)=M, R(V)=M$ and $\|U-V\| \leqslant \delta$. Moreover we have

$$
\begin{aligned}
\gamma(U) & =\inf \{\|x\| / \operatorname{dist}[(x, y), N]: x \neq 0\} \\
& =\inf \left\{\frac{\|x\|}{\inf \left\{\|(x, y)\|_{E}: y \in N\right\}}: x \neq 0\right\} \\
& =\inf \left\{\left(\inf \left\{\|(x, y)\|_{E}: y \in N\right\}\right)^{-1}:\|x\|=1\right\} \\
& =\left(\sup \left\{\inf \left\{\|(x, y)\|_{E}: y \in N\right\}:\|x\|=1\right\}\right)^{-1}
\end{aligned}
$$

Now, if $\|x\|=1$, we have $\|(x, y)\|_{E}=\max \left\{1,\|y\|, \delta^{-1}\|x-y\|\right\}$ and $\|y\| \leqslant$ $1+\|x-y\|$, from which we conclude $\gamma(U) \geqslant(1+\delta)^{-1}$.

Using Proposition 3.1, we obtain $\delta[N(U), N(V)] \leqslant(1+\delta) \delta \leqslant 2 \delta$. Note that $E / N(U)$ and $E / N(V)$ are isomorphic to $M$ and $N$, respectively.

If $P$ is $a$-costable, $N \in P$ and $\delta(M, N)<a / 2$, then $E / N(V) \in P$ and $\delta[N(U), N(V)]<a$, from which we conclude $E / N(U) \in P$, hence $M \in P$.

(2) Using the subspaces $M^{0}, N^{0} \subset X^{*}$ we construct $U, V \in L\left(E, X^{*}\right)$ as in part (1). Then, denoting by $J$ the canonical inclusion of $X$ in $X^{* *}$, we define $K:=U^{*} J$, $L:=V^{*} J \in L\left(X, E^{*}\right)$.

Analogously as in part (1) we prove $\gamma(L) \geqslant(1+\delta)^{-1}$ and $\|K-L\| \leqslant \delta$; hence $\delta[R(L), R(K)] \leqslant \delta(1+\delta) \leqslant 2 \delta$, by Proposition 3.1 .

Note that $X / M$ and $X / N$ are isomorphic to $R(U)$ and $R(V)$, respectively. The remaining part of the proof is analogous to that of (1).

In view of this result, we introduce the following definition.

Definition 2.5: $P$ is stable if it is $a$-stable for some $0<a \leqslant 1$. Equivalently, if it is $a$-costable for some $0<a \leqslant 1$.

The next result shows some properties of stable classes. 
Proposition 2.6. Let $P$ be a stable class of Banach spaces.

(1) If $M$ is a closed subspace of $X \in P$, then $M, X / M \in P$.

(2) If $X \times Y \in P$ when $X, Y \in P$, then given a closed subspace $M$ of $X$ we have $M, X / M \in P \Rightarrow X \in P$. ( $P$ has the three space property).

Proof: (1) It is enough to note that $\delta(M, X)=\delta(\{0\}, M)=0$.

(2) Suppose it is not true. Then we can find $X \notin P$ and a subspace $M$ of $X$ such that $M, X / M \in P$; hence $M \times X / M \in P$.

Now, denoting by $q$ the quotient map onto $X / M$, for every $0 \leqslant \varepsilon<1$ we define operators $Q_{\varepsilon} \in L(X \times X / M, X / M)$ by $Q_{\varepsilon}(x, y):=q x-\varepsilon y$.

In $X \times X / M$ we consider the norm $\|(x, y)\|=\|x\|+\|y\|$. We have $N\left(Q_{0}\right)=$ $M \times X / M \in P, R\left(Q_{0}\right)=X / M$ is closed, and $N\left(Q_{e}\right)=\{(\varepsilon x, q x): x \in X\}$ is isomorphic to $X$, hence $N\left(Q_{\varepsilon}\right) \notin P$ for every $0<\varepsilon<1$. However $\delta\left[N\left(Q_{e}\right), N\left(Q_{0}\right)\right] \leqslant$ $\left\|Q_{0}-Q_{\varepsilon}\right\|=\varepsilon$; hence $P$ is not stable.

Now we can show the existence of no stable classes.

EXAMPLES 2.7: (a) The classes of Banach spaces containing no copies of $c_{0}$, weakly sequentially complete spaces, and spaces with the Radon-Nikodym property are not stable: $\ell_{1}$ has a quotient isomorphic to $c_{0}$.

(b) The class of Banach spaces isomorphic to $\ell_{2}$ is not stable: In [11] a space $Z$ is given, not isomorphic to $\ell_{2}$, containing a subspace $M$ with $M$ and $Z / M$ isomorphic to $\ell_{2}$.

Unstability is related to the existence of uncomplemented subspaces, as can be seen in the following result.

Proposition 2.8. Suppose $M, N$ are subspaces of $X, N$ complemented, and let $Q$ denote a continuous projection onto $N$. If $\delta(M, N)<\|I-Q\|^{-1}$, then

(1) $M$ is isomorphic to a subspace of $N$, and

(2) $X / N$ is isomorphic to a subspace of $X / M$.

Proof: Given $x \in M$, for every $n \in N$ we have $\|Q x\| \geqslant\|x\|-\|(I-Q)(x+n)\|$. Then $\|Q x\| \geqslant\|x\|-\|(I-Q)\| \operatorname{dist}(x, N) \geqslant(1-\|I-Q\| \sigma(M, N))\|x\|$. Hence the restriction of $Q$ to $M$ defines an isomorphism into a subspace of $N$, and (1) is proved.

Moreover, the above inequality shows also that $M \cap N(Q)=\{0\}$ and $M+N(Q)=$ $Q^{-1} Q M$ is closed; hence $X / N$, which is isomorphic to $N(Q)$, is isomorphic to a subspace of $X / M$ also.

Identifying a Banach space $X$ with the corresponding subspace of the bidual $X^{* *}$, we denote by $H(X)$ the quotient space $X^{* *} / X$, and by $Q$ the quotient map from $X^{* *}$ onto $H(X)$. 
Note that for every closed subspace $M$ of $X$, the subspace $M^{\circ \circ}+X$ is closed in $X^{* *}$; hence $Q\left(M^{\circ 0}\right)$ is closed in $H(X)$.

The next result will allow us to prove some new stability results.

Lemma 2.9. Let $M$ and $N$ be closed subspaces of $X$. Then we have

$$
\delta\left[Q\left(M^{\circ \circ}\right), Q\left(N^{\circ \circ}\right)\right] \leqslant 2 \delta(M, N)
$$

ProOf: Given $m \in M^{\circ \circ}$ and $n \in N^{\circ \circ}$ we have $\|Q m-Q n\| \leqslant\|m-n\| ;$ hence $\operatorname{dist}\left[Q m, Q\left(N^{\circ \circ}\right)\right] \leqslant \operatorname{dist}\left(m, N^{\circ \circ}\right)$. If in addition we suppose $\|Q m\|=1$, then given $\varepsilon>0$ we can find $x \in S$ such that $\|m+x\|<1+\varepsilon$.

By [17, Proposition] we can find $v \in M$ such that $\|m+v\|<2(1+\varepsilon)$. Then

$$
\begin{aligned}
\operatorname{dist}\left[Q m, Q\left(N^{\circ 0}\right)\right]= & \operatorname{dist}\left[Q(m+v), Q\left(N^{\circ \circ}\right)\right] \leqslant \operatorname{dist}\left(m+v, N^{\circ \circ}\right) \\
& <2(1+\varepsilon) \delta\left(M^{\circ 0}, N^{\circ \circ}\right)=2(1+\varepsilon) \delta(M, N),
\end{aligned}
$$

since $\delta\left(M^{\circ \circ}, N^{\circ \circ}\right)=\delta(M, N)[11$, IV.2.9]. Taking the supremum in the unit sphere of $Q\left(M^{\circ}\right)$ we obtain the result of the theorem.

Theorem 2.10. If $P$ is a-stable (a-costable), then $P^{c o}:=\{X: H(X) \in P\}$ is $a / 2$-stable (a/2-costable).

ProOF: It is enough to note that given a closed subspace $M$ of $X$, we can identify (isomorphically) $Q\left(M^{\circ \circ}\right)=\left(M^{\circ \circ}+X\right) / X=M^{\circ \circ}\left(X \cap M^{\circ \circ}\right)=H(M)$, and then apply Lemma 2.9.

Recall that a Banach space $X$ is quasireflexive if $\operatorname{dim} H(X)<\infty[3]$.

Corollary 2.11. (a) The classes of quasireflexive spaces and reflexive spaces are $1 / 2$-stable and $1 / 2$-costable.

(b) The class of Banach spaces $X$ such that $H(X)$ is separable, studied in [16], is 1/4-stable and 1/4-costable.

(c) Given closed subspaces $M, N$ in $X$ with $\delta(M, N)<1 / 2$, we have

$$
\operatorname{dim} H(N)-\operatorname{dim} H(M)=\operatorname{dim} H(X / M)-\operatorname{dim} H(X / N)
$$

This is similar to the stability of the index of Fredholm operators [5].

\section{Perturbation of operators}

We shall apply the results of Section 2 to the study of perturbations of operators with closed range, using the following basic result, due to Markus [14]. We include a proof of it for the sake of completeness. 
Proposition 3.1. Let $T \in C(X, Y)$ with $R(T)$ closed, $A$ a bounded operator with $D(T) \subset D(A)$, and $S=T+A$.

(1) $\delta[N(S), N(T)] \leqslant \gamma(T)^{-1}\|S-T\|$.

(2) $\delta[R(T), \overline{R(S)}] \leqslant \gamma(T)^{-1}\|S-T\|$.

Proof: For every $x \in D(T)=D(S)$ we have $\gamma(T) \operatorname{dist}[x, N(T)] \leqslant\|T x\|$. Then, for $x \in N(S)$ we obtain $\operatorname{dist}[x, N(T)] \leqslant \gamma(T)^{-1}\|(S-T) x\|$; hence (1).

(2) Passing to a subspace of $X$, if necessary, we can suppose that $D(T)$ is dense in $X$. As $\overline{R(S)}^{\circ}=N\left(S^{*}\right), \gamma(T)=\gamma\left(T^{*}\right)\left[5\right.$, IV.1.9], and $\delta(M, N)=\delta\left(N^{\circ}, M^{\circ}\right)[11$, IV.2.9], part 2 is a consequence of (1) applied to the conjugate operator $T^{*}$.

Proposition 3.2. Let $T \in C(X, Y)$ with $R(T)$ closed, $0<a \leqslant 1$, and $A$ a bounded operator with $D(T) \subset D(A)$, and $\|A\|<a \gamma(T)$.

(1) If $P$ is a-stable and $N(T) \in P$, then $N(T+A) \in P$.

(2) If $P$ is a-costable and $Y / R(T) \in P$, then $Y / \overline{R(T+A)} \in P$.

Proof: By the above result we have $\delta[N(T+A), N(T)]<a$; hence (1) follows from the definition of $a$-stability; and analogously for (2).

Note that $R(T+K)$ is not necessarily closed.

As an application of the above perturbation theorem we give a refinement of the instability results in $[\mathbf{1}, \mathbf{7}, \mathbf{1 3}]$.

Theorem 3.3. Suppose $P$ is stable, and $M \times N \in P$ when $M \in P$ and $N$ is finite dimensional. Let $T \in C(X, Y)$ be an operator with closed range, and $K \in L(X, Y)$ a compact operator. Then

(1) $N(T) \in P \Rightarrow N(T+K) \in P$.

(2) $Y / R(T) \in P \Rightarrow Y / \overline{R(T+K)} \in P$.

Proof: (1) Since $K$ is compact we can find a finite codimensional subspace $M$ of $X$ such that $\left\|K i_{M}\right\|$ is arbitrarily small $[5,3.2 .3]$; say smaller than $a \gamma(T)$. Then $T i_{M}$ is a closed operator, $\gamma\left(T i_{M}\right) \geqslant \gamma(T)$ and $N\left(T i_{M}\right) \in P$. Hence $N\left[(T+K) i_{M}\right] \in P$ by the above theorem; and $N(T+K) \in P$, since it is the sum of $\left.N(T+K) i_{M}\right]$ and a finite dimensional subspace of $X$.

(2) This can be proved analogously, taking account of the existence of a finite dimensional subspace $N$ of $Y$ such that $\left\|q_{N} K\right\|<a \gamma(T)$, where $q_{N}$ denotes the quotient map onto $Y / N$.

\section{REFERENCES}

[1] R. Bouldin, 'The instability of non-semi-Fredholm operators under compact perturbations', J. Math. Anal. Appl. 87 (1982), 632-638. 
[2] A.L. Brown, 'On the space of subspaces of a Banach space', J. London Math. Soc. 5 (1972), 67-73.

[3] P. Civin and B. Yood, 'Quasi-reflexive Banach spaces', Proc. Amer. Math. Soc. 8 (1957), 906-911.

[4] D.P. Giesy, 'On a convexity condition in normed linear spaces', Trans. Amer. Math. Soc. 125 (1966), 114-146.

[5] S. Goldberg, Unbounded linear operators (McGraw-Hill, New York, 1966).

[6] M.A. Goldman, 'On the stability of the property of normal solvability of linear equations', (Russian), Dokl. Akad. Nauk. SSSR 100 (1955), 201-204.

[7] M. Gonzalez and V.M. Onieva, 'On the instability of non-semi-Fredholm operators under compact perturbations', J. Math. Anal. Appl. 114 (1986), 450-457.

[8] R.C. James, 'Uniformly non square Banach spaces', Ann. of Math. 80 (1964), 542-550.

[9] R.C. James, 'Some self-dual properties of normed linear spaces', Ann. of Math. Stud. 69 (1972), 159-175.

[10] R. Janz, 'Perturbation of Banach spaces', Preprint Universität Konstaz (1987).

[11] N. Kalton and N.T. Peck, 'Twisted sums of sequence spaces and the three space problem', Trans. Amer. Math. Soc. 205 (1979), 1-30.

[12] T. Kato, Perturbation Theory for linear operators (Springer-Verlag, Berlin, Heidelberg, New York, 1966).

[13] L.E. Labuschagne, 'On the instability of non-semi-Fredholm closed operators under compact perturbations with applications to ordinary differential operators', Proc. Edinburgh Math. Soc. 109A (1988), 97-108.

[14] A.S. Markus, 'On some properties of linear operators connected with the notion of gap', (Russian), Kishinev Gos. Univ. Uchen. Zap. 39 (1959), 265-272.

[15] M.I. Ostrowski, 'On properties of the opening and related closeness characterizations of Banach spaces', Amer. Math. Soc. Transl. 136 (1987), 109-119.

[16] M. Valdivia, 'On a class of Banach spaces', Studia Math. 60 (1977), 11-13.

[17] M. Valdivia, 'Banach spaces $X$ with $X^{* *}$ separable', Israel J. Math. 59 (1987), 107-111.

Departamento de Matematicas

Universidad de Cantabria

Facultad de Ciencias

39071 Santander

Spain

Departamento de Matematicas

Universidad de Cantabria

Facultad de Ciencias

39071 Santander

Spain
Departamento de Matematicas

Universidad de Oviedo

Facultad de Ciencias

33071 Oviedo

Spain 